\title{
Melt strengthening of polylactic acid and its blends: Shear and elongation rheological investigations of the forming process
}

\author{
Abderrahim Maazouz and Khalid Lamnawar
}

\author{
Abderrahim Maazouz. Université de Lyon, Ingénierie des Matériaux Polymères, IMP UMR CNRS \#5223, INSA Lyon, 20 Avenue \\ Albert Einstein, INSA de LYON, France. \\ Hassan II Academy of Science and Technology Rabat, Morocco. \\ Corresponding author: abderrahim.maazouz@insa-lyon.fr
}

Khalid Lamnawar. Université de Lyon, Ingénierie des Matériaux Polymères, IMP UMR CNRS \#5223, INSA Lyon, 20 Avenue Albert Einstein, INSA de LYON, France.

\begin{abstract}
Polylactic acid (PLA) can be a good alternative to petroleum-based polymers thanks to its organic origin and its biodegradability. This study introduces some promising routes for enhancing the processability of PLA, which presents several challenges due mainly to the poor shear and elongation properties of this biopolymer. To our knowledge, this is the first paper dedicated to an investigation of foaming and/or blown extrusion of PLA that focuses on structural, rheological and thermomechanical properties. Two main routes were selected: (i) the modification of its structural, rheological and thermomechanical properties and (ii) blending the PLA with another ductile, thermoplastic biopolymer such as poly (butylene adipateco-terephthalate) (PBAT) or polyamide (PA11). Various formulations of PLA with multifunctionalized epoxy, nucleating agents and plasticizer were prepared and characterized on the basis of their linear viscoelasticity and extensional properties. The balance of chain extension and branching was also investigated using solution viscosimetry, steric exclusion chromatography (SEC) and rheology (shear and elongation rheology). On one hand, a batch foaming process assisted by supercritical $\mathrm{CO}_{2}$ was carried out. The influence of the foaming parameters, the extent of chain modification and the contribution of crystallization to cell morphology were all evaluated. Based on these parameters, structures ranging from micro to macro-cellular-cell were obtained. On the other hand, the stability maps of blown extrusion for neat and modified PLA were established at different die temperatures. We succeeded in greatly enhancing the blown extrusion windows of PLA, achieving high blow-up ratio (BUR) and take-up ratio (TUR) values. We were able to demonstrate that faster kinetics of crystallization can also be reached for chain-extended and branched PLA formulated with adequate amounts of nucleating agents and plasticizers. Through this work, blown films with intriguing thermomechanical and mechanical properties were produced using an optimal formulation for PLA.
\end{abstract}

Keywords. Polylactic Acid, Rheology, Forming Process, Chain Extension and Branching, Blown Processing, Structure-Processing Properties Relationships

\section{Introduction}

Polylactic acid (PLA) is a polymer with many physical characteristics that make it a suitable replacement for commodity polymers [1]. In addition to being biocompatible and available from renewable resources, PLA is also strong, while demonstrating high strength, good crease retention, good grease and oil resistance and excellent aroma barrier capacities [2-5]. The high crystallinity of PLA is the reason for its high modulus and strength, but is also responsible for its brittleness and lack of toughness. These drawbacks are especially problematic in the film extrusion industry. There are, however, means of improving the flexibility and toughness of PLA, for instance by blending it with a plasticizer.

Previous laboratory studies have focused mainly on modifying the resin through branching reactions, polymer blending and charge filling [6-8]. There are also numerous reports of blending PLA with plasticizers for biomedical [9-11] and film applications [12-14]. When PLA is synthesized from corn starch, its molecular weight distribution is generally not well controlled, and the polydispersity of common commercial grades is often quite broad. This 
Melt strengthening of polylactic acid and its blends: Shear and elongation rheological ...

specificity, in addition to degradation problems [13-14] arising from the polyester nature of PLA, places the studies of this material in a different category from those focusing on classic resins [15].

A prerequisite for wider industrial use of PLA is melt strengthening, which would enlarge its processing window. The aim of the present study is therefore to investigate a commercial branching agent in its masterbatch form. The chosen pre-mixed compound is dedicated to industrial usage, for which the dosing process and the subsequent reactive extrusion need to be as simple as possible. PLA was modified through reactive extrusion with this chain brancher, and then the resulting material was characterized with regard to its molecular weight distribution (MWD) and by means of rheological analysis.

To this end, this study focuses on the forming process of PLA, through either blown extrusion or foaming. Here we present some promising routes to enhance the processability of PLA, a characteristic often compromised by the poor shear and elongation properties of this biopolymer. To our knowledge, few papers have been dedicated to an investigation of the foaming and/or blown extrusion of PLA that considers structural, rheological and thermomechanical properties [15]. For the clarity purpose of the present proceeding, only an example of results regarding the modification of structural, rheological and thermomechanical properties of PLA will be highlighted in the following section. Other illustrations of blending the PLA with PBAT or PA11 [16-17] will be presented in ESAFORM 2021 talk.

\section{Experimental part}

\subsection{Materials and reactive extrusion}

The PLA used in this study was supplied by Natureworks® (PLA 3051D®) and is referenced as an injection grade. The Joncryl ${ }^{\circledR}$ chain extender, a multifunctional ( $\mathrm{f}>9$ ) epoxide, was provided by BASF. To prevent hydrolytic degradation, PLA and Joncryl $®$ were both dried for a minimum of $4 \mathrm{~h}$ before processing. Nucleating agents were also used, as referenced in our previous patent [12]. Talc (powder <10 um), N,N'-ethylenebis(stearamide) and PEG $\left(\overline{\mathrm{M}}_{\mathrm{W}}=1450 \mathrm{~g} /\right.$ mol) were purchased from Sigma-Aldrich. The talc and N,N'-ethylenebis(stearamide) were used as nucleating agents, while PEG was used as the plasticizer. N,N'-ethylenebis(stearamide) will be referred to as EBSA in this study.

\subsection{Forming process}

(a) Foaming: commercial-grade $\mathrm{CO}_{2}$ provided by Air Liquide ${ }^{\circledR}$ was used as the blowing agent during the batch foaming process. The foaming was performed in a $300 \mathrm{~cm}^{3}$ autoclave (Model 4565C, Parr Instrument Company). The maximal working pressure was 200 bars, and the maximal temperature was $350^{\circ} \mathrm{C}$. The reactor was thermally regulated with the help of a double-wall socket with oil circulating within, which was connected to a cryo-thermostat (Phoenix C35P, Haake). In these conditions, cycles with a heating ramp of $1.3^{\circ} \mathrm{C} \cdot \mathrm{min}^{-1}$ and a cooling ramp of $1^{\circ} \mathrm{C} \cdot \mathrm{min}^{-1}$ could be set. The $\mathrm{CO}_{2}$ pressure was adjusted in the autoclave using a gaseous $\mathrm{CO}_{2}$ cylinder connected to a pneumatic gas booster (GR CRA 4AG25, Haskel Milton Roy). The pressure release was computer-controlled via a pneumatic back-pressure regulator (Tescom) allowing a maximal pressure drop rate of $2 \mathrm{MPa}^{-1}$. The autoclave was equipped with a thermocouple and a pressure transducer connected to the computer in order to monitor the temperature and pressure during all of the experiments.

In order to obtain similar foaming conditions, a basket holding four samples was designed to simultaneously foam the neat and modified PLAs. The specimens were cut from the hot-pressed plates into discs of $6 \mathrm{~mm}$ diameter and then placed in the reactor. $\mathrm{A} \mathrm{CO}_{2}$ purge was performed prior to all experiments. The first step was the saturation of the polymer with $\mathrm{CO}_{2}$ for 2 hours at $165^{\circ} \mathrm{C}$ and at pressures ranging from $9.6 \mathrm{MPa}$ up to $14.2 \mathrm{MPa}$, depending on the targeted pressure during the foaming step. This saturation period was assumed to be long enough to ensure sorption 
equilibrium in the entire sample. The second step was a thermal stabilization of the $\mathrm{CO}_{2}$ /PLA solution at the foaming temperature TF. An isothermal plateau of 20 minutes was applied before starting the polymer expansion step. Foaming was thus initiated by a pressure quench down to atmospheric pressure at a rate of about $2 \mathrm{MPa} . \mathrm{s}^{-1}$. Due to this pressure drop, the PLA matrix became supersaturated with $\mathrm{CO}_{2}$, which led to cell nucleation and growth. The foamed samples were then removed from the reactor and allowed to cool to ambient temperature.

(b) Blown extrusion: The blown extrusion relied on a tubular 25-mm diameter blow die coupled to a melt pump and twin screw extruder to process the neat and prepared formulations of PLA and Joncryl ${ }^{\circledR}$ chain extender. Molten neat and modified PLA with varying formulations was extruded using an annular die to form a tube. The temperature profile was kept constant for the different compounds, and the temperature of the tubular die was raised from $150^{\circ} \mathrm{C}$ to $180^{\circ} \mathrm{C}$. By blowing air through the die head, the tube is inflated into a thin tubular bubble and then cooled. Next, the blown film is flattened between nip rolls and pulled up by the winder. Different forms of instability are defined, and the effect of film-blowing parameters is illustrated. These parameters are the freeze-line height (FLH); the blow-up ratio (BUR), which is defined as the ratio of the final bubble diameter to the die diameter; and the take-up ratio (TUR) or draw ratio (DR), which is the ratio of the take-up velocity to the extruded material velocity at the die exit. BUR and TUR are obviously two key parameters of the film-blowing process. Increasing the values of these parameters is explicitly desirable in commercial film production. Typical BUR values in industry for HDPE fall between 1.5 and 3.5 , while TUR values range from 8 to 50. By varying the BUR, screw speed, air pressure and winder speed, films of different thicknesses between 10 to $150 \mu \mathrm{m}$ and different degrees of orientation can be manufactured.

\section{Main results and discussion}

Various formulations of PLA with multifunctionalized epoxy, nucleating agents and plasticizer were prepared and characterized on the basis of their linear viscoelasticity and extensional properties. The balance of chain extension and branching were also investigated using solution viscosimetry, steric exclusion chromatography (SEC) and rheology (relaxation spectrum, Van Gurp-Palmen plots, etc.). We pushed the challenge further by characterizing both the structural and thermomechanical properties of the PLA formulations. All of these studies provided information about the presence of branching in modified polymers, depending on the amount of Joncryl ${ }^{\circledR}$ used and the reaction time. The resulting plots were characteristic of long-chain branched polymers. Indeed, they exhibited the typical features of a mixture of linear and randomly branched polymers.

On one hand, a batch foaming process assisted by supercritical $\mathrm{CO}_{2}$ was carried out following a complete physicochemical, rheological and thermal characterization study. The influence of the foaming parameters, the extent of chain modification and the contribution of crystallization to cell morphology were all evaluated [11]. Based on these parameters, structures ranging from micro to macro-cellular-cell were obtained (Figure 1). 


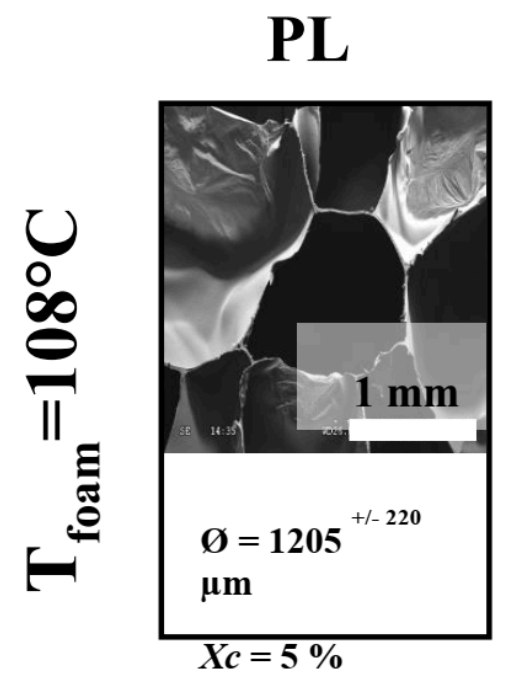

PL

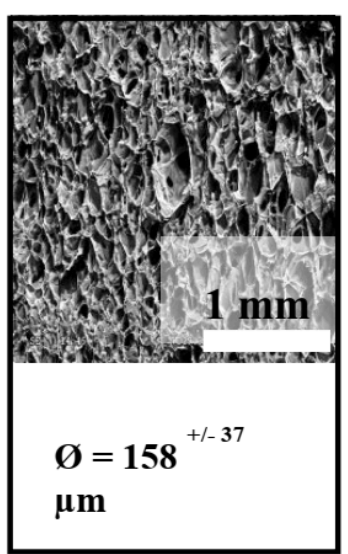

$X c=26,5 \%$
PL

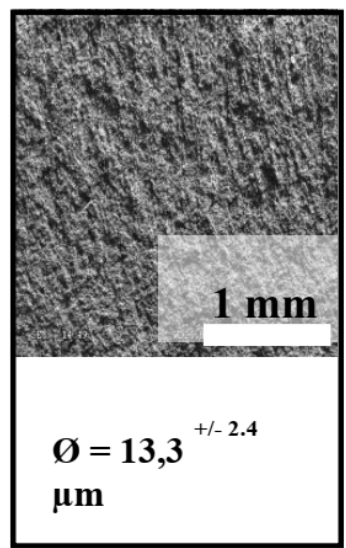

$X c=31,6 \%$
PL

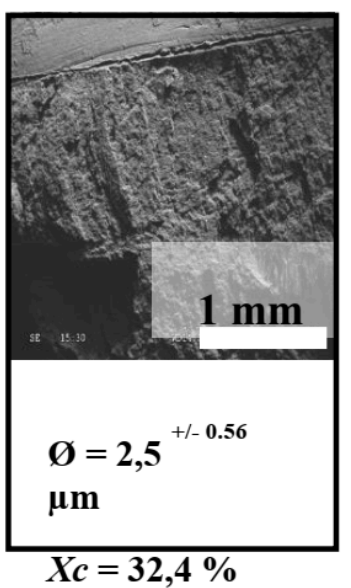

Figure 1 - Examples of cellular morphology of neat and modified PLA obtained by supercritical $\mathrm{CO}_{2}$ fluid ( $\mathrm{X}_{\mathbf{C}}$ corresponds to the PLA crystallinity amount and $\emptyset$ is the average cellular size)

On the other hand, the stability maps of blown extrusion or neat and modified PLA were established at different die temperatures. We succeeded in greatly enhancing the blown extrusion windows of PLA, achieving high BUR and TUR values (Figure 2). 
(a)
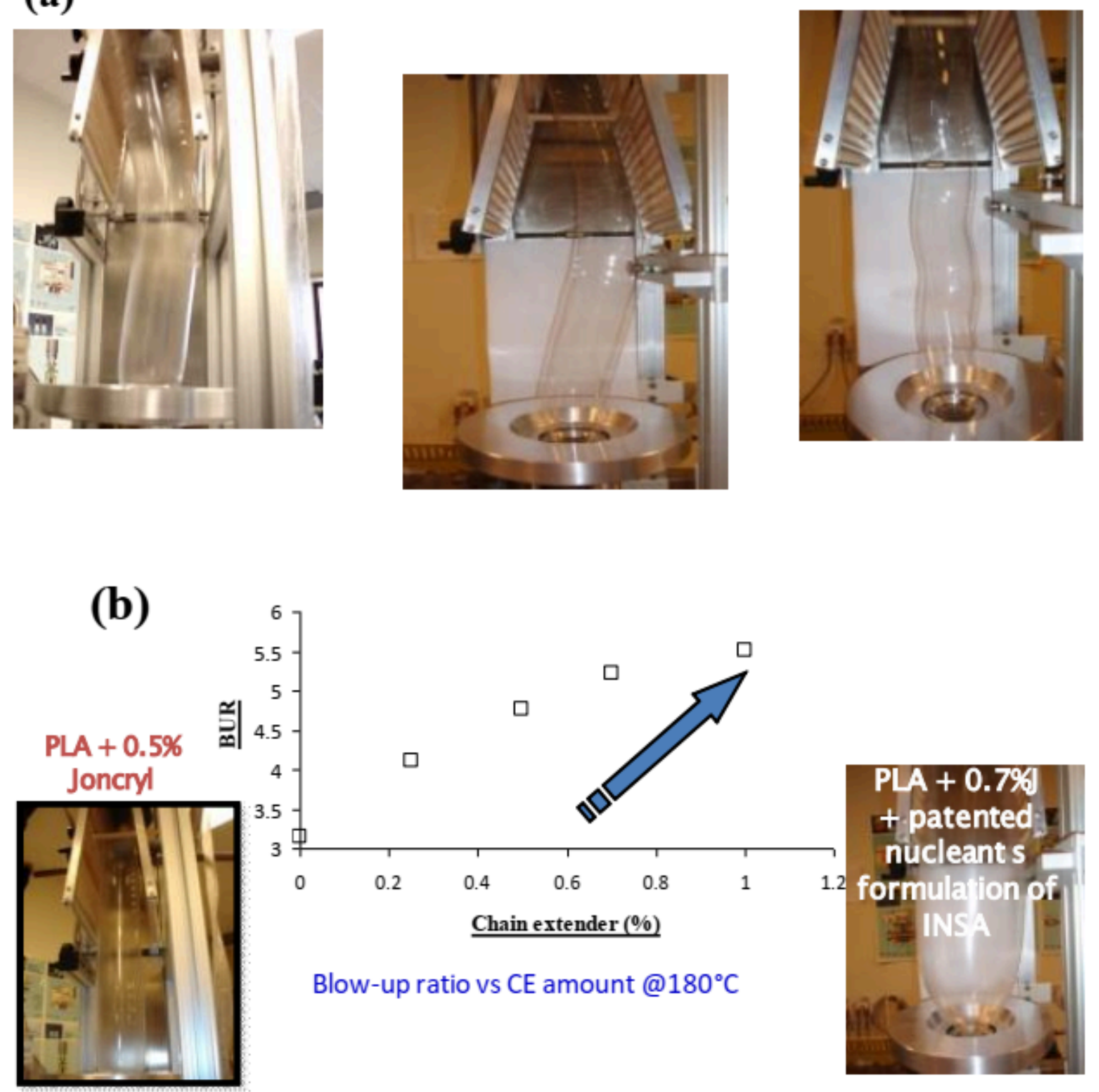

Figure 2 - Improvement of the blown extrusion properties of modified PLA: (a) neat PLA with defects, and (b) stable blown films of modified PLA.

Furthermore, the stability maps of processed blends were established. When compared to a PLA/Joncryl $囚$ blend, the ternary system (including Talc (powder $<10 \mathrm{um}$ ), N,N'-ethylenebis(stearamide) and PEG) leads to a clear enlargement of the processing window, with bubbles obtained at higher BUR ratios. Of most interest is the significant increase in the maximal value for stable BUR ratios, climbing from 7 to 12 at high TUR. The maximal BUR ratio that could possibly be attained is higher than this, but the equipment used in our study had reached its limits. This surprising behavior is consistent with the effects of chain extension branching and the role of nucleating agents and plasticizers. The blown bubbles also showed a translucent aspect that can be associated with a semi-crystalline structure characterized using DSC, WAXS and FTIR as detailed in [12-17]. By using a ternary system with a chain extension/branching reaction, faster crystallization kinetics for PLA were achieved. This can induce crystallization during the blowing process at higher BUR and TUR ratios (e.g., stretching and orientation of chains). These results are interesting enough to warrant a thorough analysis of the microstructural properties of such blown-processed films, which is a study that our team 
Melt strengthening of polylactic acid and its blends: Shear and elongation rheological ...

is currently undertaking. Furthermore, we were able to demonstrate that faster kinetics of crystallization can also be reached for chain-extended and branched PLA formulated with adequate amounts of nucleating agents and plasticizers. Induced crystallization during the process was also demonstrated. Through this work, blown films with intriguing thermomechanical and mechanical properties have been produced using an optimal formulation for PLA and its blends [14-17].

\section{Conclusion}

Polylactic acid (PLA) was modified through reactive extrusion, and chain branching was achieved with the help of an epoxide additive. An optimized processing temperature was identified as a key parameter to obtain the desired efficiency of the reaction, because it reduces the competition between chain extension and thermal chain scission.

Chain branching drastically modified the PLA structure by shifting the molecular weight distribution from a monomodal to a bimodal population, and this new chain distribution had an enlarging effect on the relaxation time spectrum. Moreover, the melt rheology of the materials was influenced by these molecular couplings. On one hand, the low shear viscosity could be drastically improved by increasing the amount of branching agent used. On the other hand, converging values were obtained for high shear rates. Thus, the melt obviously became stronger with increasing epoxide content. The thermal properties of the modified PLA materials were found to diverge from those of neat PLA. For instance, crystallization nucleation was strongly enhanced when branching reactions were performed. This phenomenon induced a kinetic improvement but altered the final crystallinity, which was found to become lowered when the branching level was increased. Finally, the branched materials featured a broader crystallization temperature window compared to that of the neat PLA.

On one hand, the foaming ability of neat PLA was enhanced by chain extension through reactive extrusion. The resulting rheological improvements were found to be key parameters for enlarging the processing window over foaming temperatures. Elsewhere, the crystallization behavior under supercritical $\mathrm{CO}_{2}$ was found to vary drastically from neat to highly chain-extended PLAs. This is of special importance for the micro-scaled foams obtained at low foaming temperatures with the chain-extended PLAs. In this case, the foaming seems to be governed by nucleation.

On the other hand, both the processing stability and the microstuctural properties of the blown PLA-based film were improved. As demonstrated herein, the blown extrusion of various grades of the neat PLA was limited, so a proper correlation between melt shear and extensional rheological properties is very important to get a better handle on this process. Our challenge was to make this process stable at higher melting temperatures with the highest BUR and TUR ratios [16-17] sustainable, by blending PLA with appropriate additives. It was clearly demonstrated that the addition of multifunctionalized epoxide (Joncryl $\AA$ ) has a direct influence on the rheological behavior of PLA. Thermomechanical properties of PLA and the PLA-based optimized blends were also investigated, confirming the presence of a crystalline structure induced during processing. The mechanical properties of the PLA-based optimized blend showed a more flexible material with ductile behavior compared to the brittle neat PLA.

\section{Bibliography}

[1] M. Vert, G. Schwarch, J. Coudane, Present and future of PLA polymers. J. Macromol. Sci., Part A. Pure Appl. Chem. 32 (1995) 787-796.

[2] J. Lunt, Large-scale production, properties and commercial applications of polylactic acid polymers. Polym. Degrad. Stabil. 59 (1998) 145-152.

[3] R. H. Wehrenberg, Lactic acid polymers: strong, degradable thermoplastics. Mater. Eng.94 (1981) 63-66. 
[4] R. E. Drumright, P. R. Gruber, D. E. Henton, Polylactic Acid Technology. Adv. Mater. 12 (2000) 1841 - 1846.

[5] E.S. Lipinsky, R. G. Sinclair, Is lactic acid a commodity chemical. Chem. Eng. Prog. 82(1986) 26-32.

[6] J. R. Dorgan, J. S. Williams, D.N. Lewis, Melt rheology of poly(lactic acid): Entanglement and chain architecture effects. J. Rheol. 43 (1999) 1141-1155.

[7] Z. F. Zhou, G. Q. Huang, W. B. Xu, F. M. Ren, Chain extension and branching of poly(L-lactic acid) produced by reaction with a DGEBA-based epoxy resin. eXPRESS Polym. Lett. 1 (2007) 734-739.

[8] A. L. Arraiza, J.R. Sarasua, J. Verdu, X. Colin, Rheological behavior and modeling of thermal degradation of poly(ecaprolactone) and poly(L-Lactide). Polymer processing XXII 5(2007) 389-394.

[9] J. D. Campbell, , J. A. Debling, D. J. DeYoung, I. Giannakitsas, D. R. Hellwig, D. D. Schatz, F. Teymour, M. A. Villalobos, Process for producing polymers by free radical polymerization and condensation reaction, and apparatus and products related thereto. U.S. Patent 6, 346, 590. (2002).

[10] M. Villalobos, A. Awojulu, T. Greeley, G. Turco, G. Deeter, Oligomeric chain extenders for economic reprocessing and recycling of condensation plastics. Energy 31 (2006) 3227-3234.

[11] Y-M. Corre, A. Maazouz, J. Duchet, J. Reignier, Batch foaming of chain extended PLA with supercritical CO2: Influence of the rheological properties and the process parameters on the cellular structure. J. of Supercritical Fluids58(2011) 177- 188.

[12] A. Maazouz, K. Lamnawar, B. Mallet, F.R. Patent 2941702 (A1), (2010)

[13] B. Mallet; K. Lamnawar; A. Maazouz, Polymer Engineering and Science. 54(4), (2014) 840-85,

[14] K. Lamnawar, A. Maazouz, G. Cabrera, R. Al-Itry, Interfacial Tension Properties in Biopolymer Blends: From Deformed Drop Retraction Method (DDRM) to Shear and Elongation Rheology-Application to Blown Film Extrusion. International Polymer Processing Vol. 33, 3 (2018)411-424

[15] M. Abid, B. Mallet, K. Lamnawar, A. Maazouz, Crystallization of Poly (Lactic Acid), PLA: Effect of Nucleating Agents and Structure-Properties Relationships, Journal of Composites and Biodegradable Polymers, 6, (2018) 34-46 (Online 2018, E-ISSN: 2312-5470/18),

[16] F. Walha, K. Lamnawar, A. Maazouz, M. Jaziri, Preparation and characterization of Bio-sourced Blends based on Poly (lactic acid) and Polyamide 11: Structure-properties relationships and Enhancement of film blowing processability. Advances in Polymer Technology, Volume: 37 Issue: 6 (2018) 2061-2074

[17] R. Al-Itry, K. Lamnawar, A. Maazouz, Biopolymer Blends Based on Poly (lactic acid): Shear and Elongation Rheology/Structure/Blowing Process Relationships. Polymers, 7 (2015), 939-962.

PDF automatically generated on 2021-05-20 19:59:08

Article url: https://popups.uliege.be/esaform21/index.php?id=748

published by ULiège Library in Open Access under the terms and conditions of the CC-BY License (https://creativecommons.org/licenses/by/4.0) 